\title{
BMJ Open Linkage into care among newly diagnosed HIV-positive individuals tested through outreach and facility- based HIV testing models in Mbeya, Tanzania: a prospective mixed-method cohort study
}

\author{
Erica Samson Sanga, ${ }^{1,2}$ Wondwossen Lerebo, ${ }^{2,3}$ Adiel K Mushi, ${ }^{4}$ Petra Clowes, ${ }^{1,5}$ \\ Willyhelmina Olomi, ${ }^{1}$ Leonard Maboko, ${ }^{1}$ Christina Zarowsky ${ }^{2,6}$
}

To cite: Sanga ES, Lerebo W, Mushi AK, et al. Linkage into care among newly diagnosed HIV-positive individuals tested through outreach and facility-based HIV testing models in Mbeya, Tanzania: a prospective mixed-method cohort study. BMJ Open 2017:7: 013733 .

doi:10.1136/bmjopen-2016013733

- Prepublication history for this paper is available online To view these files please visit the journal online (http://dx.doi.org/10.1136/ bmjopen-2016-013733).

Received 20 August 2016 Revised 18 February 2017 Accepted 27 February 2017

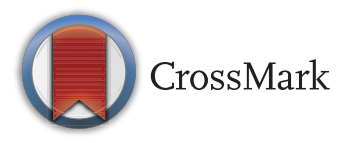

For numbered affiliations see end of article.

Correspondence to Erica Samson Sanga; esanga@nimr-mmrc.org

\section{ABSTRACT}

Objective: Linkage to care is the bridge between HIV testing and HIV treatment, care and support. In Tanzania, mobile testing aims to address historically low testing rates. Linkage to care was reported at $14 \%$ in 2009 and $28 \%$ in 2014 . The study compares linkage to care of HIV-positive individuals tested at mobile/ outreach versus public health facility-based services within the first 6 months of HIV diagnosis.

Setting: Rural communities in four districts of Mbeya Region, Tanzania.

Participants: A total of 1012 newly diagnosed HIVpositive adults from 16 testing facilities were enrolled into a two-armed cohort and followed for 6 months between August 2014 and July 2015. 840 (83\%) participants completed the study.

Main outcome measures: We compared the ratios and time variance in linkage to care using the KaplanMeier estimator and Log rank tests. Cox proportional hazards regression models to evaluate factors associated with time variance in linkage.

Results: At the end of 6 months, $78 \%$ of all respondents had linked into care, with differences across testing models. $84 \%$ ( $\mathrm{Cl} 81 \%$ to $87 \%, \mathrm{n}=512$ ) of individuals tested at facility-based site were linked to care compared to $69 \%(\mathrm{Cl} 65 \%$ to $74 \%, \mathrm{n}=281$ ) of individuals tested at mobile/outreach. The median time to linkage was 1 day (IQR: 1-7.5) for facility-based site and 6 days (IQR: $3-11)$ for mobile/outreach sites. Participants tested at facility-based site were $78 \%$ more likely to link than those tested at mobile/outreach when other variables were controlled (AHR=1.78; $95 \% \mathrm{Cl}$ 1.52 to 2.07). HIV status disclosure to family/relatives was significantly associated with linkage to care (AHR=2.64; 95\% Cl 2.05 to 3.39).

Conclusions: Linkage to care after testing HIV positive in rural Tanzania has increased markedly since 2014, across testing models. Individuals tested at facility-based sites linked in significantly higher proportion and modestly sooner than mobile/outreach

\section{Strengths and limitations of this study}

Prospective adequately powered cohort study.

- Participants followed up for 6 months, with good retention $(83 \%)$

- Some participants may have moved elsewhere during the study and may have accessed care elsewhere; this warrants further investigation.

- Retention was higher in facility-testing arm $(87 \%)$ than in mobile-testing arm $(76 \%)$.

- Participant tracking might have enhanced linkage to care.

tested individuals. Mobile/outreach testing models bring HIV testing services closer to people. Strategies to improve linkage from mobile/outreach models are needed.

\section{INTRODUCTION}

HIV remains a major burden in Sub-Saharan Africa (SSA), with 790000 deaths associated with HIV in 2014. ${ }^{1}$ Despite the high prevalence and the increasing numbers of people living with HIV in need of highly active antiretroviral therapy (HAART), timely linkage to care is generally poor across $\mathrm{SSA} .^{2}{ }^{3}$ The Mbeya Region is among the three regions in Tanzania with the highest HIV prevalence, with an average of $9 \%$ compared to the national average of $5.1 \%,{ }^{4}$ and AIDS-related deaths are among the three leading causes of death in the area. ${ }^{5}$

Linkage to care is the bridge between HIV testing and HIV treatment, care and support. ${ }^{4}$ Timely HIV diagnosis and effective linkage into care and treatment are keys to 
improved outcomes. ${ }^{7} 8$ All individuals diagnosed HIV positive must be linked to HIV care and treatment even if local treatment guidelines do not indicate that a person should be started on antiretroviral therapy immediately. ${ }^{9} \mathrm{CD} 4$ cell count, HIV staging and evaluation of the client's need for antiretroviral therapy (ART) initiation need to be performed immediately. The Ministry of Health and Social Welfare in Tanzania guideline for initiation of $\mathrm{ART}^{\mathrm{i}}$ is a CD4 count $\leq 500$ cells; ${ }^{10}$ however, during the period of this study, the actual cut-off point for ART initiation was a CD4 count of 350. The importance of linkage to care during HIV counselling and testing has been well advocated in Tanzania; however, the available literature indicates that linkage to care after testing HIV positive is still low, with only $14 \%$ linkage at 4 months reported in a 2009 study, and only $23 \%$ in Ifakara and $28 \%$ in Mbeya Region in $2014 .{ }^{11-13}$ Low or delayed linkage to care leads to failure of HIV-positive individuals to benefit from HIV care. Hence, efforts are hampered to improve coverage for HIV care and treatment services, thus resulting in increased risk of HIV transmission to others. ${ }^{414}$ Linkage to care remains at suboptimal levels in the country due to barriers such as lack of understanding of the importance of care regardless of disease stage, distance from the clinic and transport costs. ${ }^{111516}$ Fear of stigma related to HIV, failure to disclose HIV status, being asymptomatic at the time of diagnosis and negative attitudes of healthcare providers are other factors reported to interfere with linkage to HIV care. ${ }^{17-19}$

Mobile and outreach testing sites have been introduced in Tanzania, reflecting an increasing interest in providing early detection of HIV and subsequent care and support in the hard to reach populations and remote areas. ${ }^{16}{ }^{20}$ Most government health facilities in Mbeya Region (the site of this study) offer providerinitiated testing and counselling (PITC) and voluntary counselling services, but only about $21 \%$ also offer HIV care and treatment services. ${ }^{13}$ On the other hand mobile and outreach services, operated mostly by nongovernmental organisations (NGOs), usually offer only voluntary counselling and testing (VCT) services. $^{13}$ These sites do not offer HIV care services, with the exception of the research mobile laboratory operating under the Mbeya Medical Research Centre (MMRC) that offers CD4 testing on site. Clients that test HIV-positive must then go to facility-based sites for registration and other procedures for HIV care and treatment. $^{6} 13$

There has been little research on overall linkage to care in Tanzania, and none to the best of our knowledge on whether linkage to care differs between clients diagnosed at mobile/outreach sites compared to health facilities, or on factors facilitating or inhibiting successful linkage to care between these two models of service

${ }^{\mathrm{i}}$ First line ART in Tanzania is Tenofovir, Lamivudine and Efevirenz. ${ }^{10}$ delivery. ${ }^{21} 22$ These differences may occur at the patient level, at service provider level, at the facility level or at the level of the health system as a whole. For example, factors enhancing access to testing, such as dedicated outreach staff, may enhance linkage to care for those testing in mobile/outreach facilities, while other factors such as geographic distance between patients' homes and testing sites and treatment sites, weak referral systems and lack of structural links between testing and treatment sites may lead to disconnects between testing and care. ${ }^{11} 1623$

One South African study found that individuals testing at mobile services were $33 \%$ less likely to undergo CD4 testing than individuals testing at static clinic services, and only $10 \%$ of mobile testers were successfully linked into care versus $72 \%$ of clinic testers; ${ }^{21}$ however, in South Africa nearly all health facilities now offer treatment, care and support. Hence, findings about differences between mobile and facility-based testing and subsequent linkage to care may not be directly transferable to Tanzania, where testing and care are not always available as a 'one stop shop'. Active referral or selfreferrals are therefore more common in Tanzanian situations.

Mbeya Region has a total of 312 health facilities where clients can receive testing and counselling (HTC) services through recommended approaches; however, only 68 facilities (21.7\%) offer HIV care and treatment service. ${ }^{13}$ At least two outreach partners or NGOs offer HIV counselling and testing in each district of the Mbeya Region. The Mbeya Medical Research Centre MMRC mobile laboratory, also known as the Mobile Diagnostic and Training Centre (MDTC), has been offering CD4 count tests at point of care ${ }^{5}$ since 2009, covering between 8 and 12 sites every 3 months. Available statistics from the Mbeya Regional AIDS Control program (MRACP) ${ }^{6}$ suggest that more people undergo HIV testing at mobile/outreach HIV testing services (56\%) compared to facility-based services (44\%); however, only about $28 \%$ of all people tested were linked into HIV care. ${ }^{21}$ An earlier study conducted in Mwanza reported that despite increased testing opportunities only $14 \%$ of newly diagnosed patients had linked into care 4 months after HIV diagnosis. ${ }^{11}$ Another study on linkage to care conducted in Ifakara showed a linkage of about $23 \%$, indicating that linkage to care is a challenge in Tanzania. ${ }^{12}$

This article reports new findings on linkage to care and compares the outcomes of linkage and time to linkage into care for individuals tested HIV positive at mobile/outreach sites, versus individuals tested HIV positive at facility-based services over the first 6 months after diagnosis in rural parts of the Mbeya Region. The findings of this study are expected to inform policymakers and other stakeholders in the Tanzanian healthcare system on the optimisation of HIV testing and immediate linkage to care, an issue of critical importance for timely initiation of antiretroviral therapy. 


\section{STUDY DESIGN AND METHODS}

This was a prospective mixed-method cohort study of 1012 adults who tested HIV positive recruited into a two-armed cohort (health facility-based vs mobile/ outreach HIV testing sites). The study participants were followed for 6 months to gather quantitative and qualitative information on linkage to care since diagnosis.

\section{Study setting}

The study population comprises rural communities in four of the then eight districts of the Mbeya Region in 2014. In 2012, the Mbeya Region had a population of 2707410 with $52 \%$ women and $48 \%$ men. ${ }^{24}$ The four study districts were selected to include high HIV prevalence areas and hard-to-reach populations. Two districts (Kyela and Mbozi) are along the highways and have borders with Zambia and/or Malawi. The population in Kyela district was 221495 in 2012, while Mbozi had 446339 residents. High population mobility associated with cross-border business and social interactions is thought to pose challenges to linkage to and continuity of care in these districts. The other two districts (Mbeya Rural and Chunya) have a larger proportion of residents who live $10 \mathrm{~km}$ or more from a health facility. The population in these remote districts was 305319 and 290 478, respectively. ${ }^{24}$ The HIV prevalence among people tested for HIV in 2014 in the selected districts were Mbeya Rural $13.0 \%$, Chunya 9.2\%, Kyela 9.2\%, and Mbozi $8.7 \%$.

All public and mission health facility-based and outreach/mobile sites in the selected districts were listed. A total of 27 health facility and 4 mobile/outreach sites were listed in Mbeya rural, 20 health facility-based and 4 mobile/outreach sites in Chunya district, 14 facilitybased and 5 mobile/outreach sites in Kyela district and 29 health facility-based and 5 mobile/outreach sites in Mbozi district. Four sites in each district (two facilitybased and two mobile/outreach sites) were randomly selected from the list in each district using a table of random numbers. The eight facility-based sites selected had a care and treatment centre (CTC) within the facility. Sites had different arrangements for the first step of linkage to care, registration: in some facilities, registration was possible on the same day as testing, while other facilities had chosen a single day or two per week for newly diagnosed clients to register into HIV care. None of the mobile/outreach sites offered CTC services; they had to refer their clients to the closest CTC for further management (HIV staging, laboratory test, ART initiation, etc). The mobile site from MMRC was offering CD4 tests at the point of care, but still had to refer newly diagnosed clients, already with their CD4 results to nearby HIV care clinic or CTCs for registration and continuation of care.

\section{Sampling}

The sampling strategy for testing sites is described above. The sampling framework for the cohort comprised all adults above 18 years receiving HIV testing at facility-based and mobile/outreach sites in the four study districts of Mbeya Region. The sample size was calculated using Epi Info software with a CI of 95\% and power of $90 \%$, assuming that the two study groups would have the same number of participants. Thirty per cent of individuals tested through mobile/outreach services and $41 \%$ of individuals tested at facility-based services were expected to link to HIV care. The estimated sample size was 828; we adjusted this sample size to account for possible dropouts and non-responders $(10 \%)$ resulting in a total estimated sample size of 900 participants.

\section{Data collection procedures}

Prior to data collection at clinic, the research team briefed the nurse counsellors at study sites on the study objectives and procedures. In turn, these nurse counsellors introduced the research team to clients. Interested individuals were invited in a private room for detailed explanation, informed consent process and agreement on a convenient time and place for questionnaire administration. Initial data were collected between August and December 2014. Follow-up questionnaire administration continued until June 2015. Eight of 1020 individuals who were approached for participating during data collection were not enrolled in the study because two of them were seriously sick and needed hospital admission, three were planning to move out of Mbeya to their home villages after receiving the results and the other three did not come back for enrolment and interviews within 7 days of testing and we were unable to track them. Research assistants who underwent 2 days of training on informed consent and data collection procedures did data collection.

\section{Outcome measures}

The key outcome was the proportion of participants successfully linked to HIV CTC across the sample and in each arm of the cohort. In this study, 'facility-based sites' refer to fixed or static facilities such as hospitals, health centres and dispensaries while 'mobile/outreach sites' means all outreach HIV testing services, including campaigns, mobile testing clinics, home visits or special event testing services.

The operational definition for linkage to care in this study is that a newly diagnosed individual has reported to a CTC, completed the registration process and has been provided with a CTC registration number and clinic card. This definition of linkage to care is based on Rosen and Fox $^{25}$ and the National AIDS Control Programme in Tanzania; ${ }^{20}$ it was chosen to allow comparison with earlier studies of linkage to care.

This paper reports on preliminary outcomes for which a structured questionnaire was administered to respondents at enrolment, at 3 months and at 6 months to ascertain time to linkage into HIV care and to explore factors related to linkage to care. Information collected 
at enrolment included demographic data, date of HIV testing, reasons for testing, plans for linkage into care and plans for disclosure of HIV status to any family member, other relative or friend. All baseline information was self-reported by participants. In follow-up interviews at 3 and 6 months, we asked about registration/ linkage into care, CD4 count testing, ART status and results disclosure status. At these follow-up interviews, we also reviewed the participants' clinic card to verify the reported dates of linkage, ART initiation and CD4 count results.

\section{Data analysis}

Quantitative data from sites were recorded, cleaned and analysed using Stata V.13 (College Station, Texas, USA). Descriptive analysis methods were used to present the characteristics of participants. Categorical data were presented using frequencies and percentage, while quantitative data were presented using the measure of central tendency and measure of dispersion. Cross-tabulation was used to show the distribution of study participant by testing site. We compared the ratios and time variance in linkage to care using the Kaplan-Meier estimator and Log rank tests. Cox proportional hazards regression models were used to evaluate the factors associated with time variance in linkage to care. Statistical significance was declared at $p$ values $<0.05$ for the entire analysis.

\section{Ethical considerations}

The study was approved by the University of Western Cape (UWC) Senate Research Committee, the Mbeya Medical Research Centre, the Mbeya Medical Research Ethics Committee (MMREC) and the National Health Research Ethics Sub-Committee (NatHREC) under the Tanzanian National Institute of Medical Research (NIMR). Participation was voluntary, and it was explained to participants that they were free to withdraw from the study at any time without negative consequences. Volunteers were provided with an information sheet containing all details about the study. They signed an informed consent, and confidentiality procedures were observed.

\section{RESULTS}

Participant characteristics and comparison between facility-based and mobile-based testing models

The cohort of 1012 HIV-positive individuals included $58.5 \%$ female participants (56\% facility; $61 \%$ mobile), with a mean age of 35.8 years (SD 10.5) for facility-based and 35.3 years (SD 10.0) for mobile/outreach participants. By the end of 6 months follow-up overall $83 \%$ of participants were still active in the study, $87 \%$ in the facility-based arm and $76 \%$ in the mobile/outreach arm $(\mathrm{p}<0.0001)$. In both testing models, about $60 \%$ of participants were married and more than $80 \%$ of participants were self-employed with small-scale farming or petty businesses. A detailed listing of the patient characteristics is presented in table 1. Age, gender, level of education and occupation were not statistically different between the two testing models, while statistical differences in marital status, means of transport, time to reach clinic, income and time to linkage were observed after $\chi^{2}$ analysis.

\section{Linkage to care at 6 months}

At 6 months, $78 \%$ of enrolled participants were linked into care across both arms. Eighty-four per cent $(95 \%$ CI $0.81 \%$ to $0.87 \%$ ) of participants tested at the facilitybased sites were linked into care within the first 6 months of HIV diagnosis, compared to $69 \%$ (95\% CI $0.65 \%$ to $0.74 \%$ ) from the mobile/outreach-tested group (figure 1). The interval from the day of HIV testing to the day of registration at a CTC was compared between participants who tested at a health facility and those tested through a mobile/outreach model. The median time to linkage was 1 day (IQR 1-7.5 days) for those who tested at a health facility and 6 days (IQR 3-11 days) for those who tested through any mobile/ outreach model.

\section{CD4 cell counts facility-based sites and mobile sites}

Of the 793 clients linked into care, 512 (64.5\%) tested in facility-based sites and $281(35.4 \%)$ tested in mobile/ outreach sites. Most of the clients $(\mathrm{n}=774,97.6 \%)$ had a recorded CD4 count. The median CD4 count among participants who tested in facility-based sites was 220 (IQR: 114-382), while among those tested in mobile/ outreach sites the median CD4 count of 255 (IQR: 174394). Student's t-test showed no statistical difference in $\mathrm{CD} 4$ count at the point of linkage to care between the two testing models $(\mathrm{p}=0.49)$.

\section{Time to linkage facility-based and mobile sites}

The time to linkage (registration) was significantly shorter in the facility tested group, compared to the mobile/outreach tested group $(\mathrm{p}<0.001)$ (figure 2$)$. Log rank test showed that there was a significant difference between the two groups $(\mathrm{p}<0.001)$. Sensitivity analysis was carried out on the 840 participants who were successfully followed for 6 months. Cox regression analysis revealed that a person tested at a facility-based site increased the 'risk' of linkage by $61 \%$ (adjusted hazard ratio (AHR) $=1.61 ; 95 \%$ CI 1.39 to 1.85 ) compared to persons tested at mobile sites. The log-rank test found a significant difference between the two groups $(\mathrm{p}<0.001)$.

\section{Linkage from mobile sites with point of care CD4 test versus no CD4 test}

Of the 405 participants testing at mobile/outreach sites, $182(44.94 \%)$ individuals had tested for HIV at the MMRC mobile site, where CD4 testing was offered at the point of testing, but no registration or ART was provided. A total of $223(55.06 \%)$ individuals tested for HIV at mobile/outreach sites without the availability of 
Table 1 Background characteristics of study participants by site

\begin{tabular}{|c|c|c|c|c|}
\hline Variable & Facility based & Mobile & $\mathbf{N}$ & p Value \\
\hline \multicolumn{5}{|l|}{ Gender } \\
\hline Male & 265 (43.66) & $157(38.77)$ & 422 (41.70) & \multirow[t]{2}{*}{0.122} \\
\hline Female & 342 (56.34) & $248(61.23)$ & $590(58.30)$ & \\
\hline Age, mean (SD) & $35.8(10.5)$ & $35.3(10)$ & & 0.9 \\
\hline \multicolumn{5}{|l|}{ Marital status } \\
\hline Single & 78 (12.85) & $48(11.85)$ & $126(12.45)$ & \multirow{5}{*}{0.002} \\
\hline Married & 361 (59.47) & 252 (62.22) & $613(60.57)$ & \\
\hline Separated & $82(13.51)$ & $37(9.14)$ & 119 (11.76) & \\
\hline Divorced & $13(2.14)$ & $26(6.42)$ & 39 (3.85) & \\
\hline Widower & 73 (12.03) & $42(10.37)$ & 115 (11.36) & \\
\hline \multicolumn{5}{|l|}{ Level of education } \\
\hline None & 104 (17.13) & $81(20.00)$ & 185 (18.28) & \multirow{4}{*}{0.4} \\
\hline Primary & 470 (77.43) & 299 (73.83) & 769 (75.99) & \\
\hline Secondary & $29(4.78)$ & $24(5.93)$ & $53(5.24)$ & \\
\hline Vocational & $4(0.66)$ & $1(0.25)$ & $5(0.49)$ & \\
\hline \multicolumn{5}{|l|}{ Main occupation } \\
\hline Unemployed & $28(4.61)$ & $15(3.70)$ & $43(4.25)$ & \multirow{6}{*}{0.23} \\
\hline Student & $18(2.97)$ & $3(0.74)$ & 21 (2.08) & \\
\hline Driver & $9(1.48)$ & $5(1.23)$ & $14(1.38)$ & \\
\hline Employed & $18(2.97)$ & $11(2.72)$ & $29(2.87)$ & \\
\hline Self- employed & $530(87.31)$ & 369 (91.11) & 899 (88.83) & \\
\hline Other & $4(0.66)$ & $2(0.49)$ & $6(0.59)$ & \\
\hline \multicolumn{5}{|l|}{ Means of transport } \\
\hline Walking & $163(26.85)$ & 200 (49.38) & 363 (35.87) & \multirow{5}{*}{$p<0.0001$} \\
\hline Bicycle & 93 (15.32) & 77 (19.01) & $170(16.80)$ & \\
\hline Motor cycle & $143(23.56)$ & $71(17.53)$ & $214(21.15)$ & \\
\hline Public transport & 201 (33.11) & $55(13.58)$ & $256(25.30)$ & \\
\hline Private car & $7(1.15)$ & $2(0.49)$ & $9(0.89)$ & \\
\hline \multicolumn{5}{|l|}{ Time to reach clinic (hours) } \\
\hline$<1$ & $397(65.40)$ & $295(72.84)$ & $692(68.38)$ & \multirow{4}{*}{0.004} \\
\hline $1-2$ & $157(25.86)$ & $76(18.77)$ & $233(23.02)$ & \\
\hline $2-5$ & $50(8.24)$ & $26(6.42)$ & $76(7.51)$ & \\
\hline$>5$ & $3(0.49)$ & $8(1.98)$ & $11(1.09)$ & \\
\hline Time to linkage, median (IQR) & $1(1-7.5)$ & $6(3-11)$ & & $p<0.0001$ \\
\hline \multicolumn{5}{|l|}{ Income (Tsh) } \\
\hline$<100000$ & $497(81.88)$ & 320 (79.01) & $817(80.73)$ & \multirow{6}{*}{0.0006} \\
\hline $100000-500000$ & $39(6.43)$ & $56(13.83)$ & $95(9.39)$ & \\
\hline $500000-1000000$ & $3(0.49)$ & $2(0.49)$ & $5(0.49)$ & \\
\hline$>1000000$ & $0(0.00)$ & $1(0.25)$ & $1(0.1)$ & \\
\hline NA & $52(8.57)$ & $21(5.19)$ & $73(7.21)$ & \\
\hline Refused to answer & $16(2.64)$ & $5(1.23)$ & $21(2.08)$ & \\
\hline
\end{tabular}

CD4 tests, registration and ART. A total of $66.5 \%$ of study participants testing for HIV with an immediate CD4 test and $72 \%$ of those testing at a site without CD4 test were linked into care within the first 6 months; however, this difference was not statistically significant.

\section{Factors associated with time to linkage}

Bivariate Cox regression showed that there were several factors associated with hazard of time to linkage, and multivariate Cox regression analysis revealed that a person tested at facility-based increase the risk of linkage by $78 \%$ (AHR $=1.78 ; 95 \%$ CI $1.52 \%$ to $2.07 \%$ ) compared with persons tested at mobile centre when other variables were controlled. Disclosure of HIV status to partners, family, a relative or a friend was found to be a significant factor associated with two and a half times increased risk of linkage to care $(\mathrm{AHR}=2.64 ; 95 \%$ CI 2.05 to 3.39). Of the participants whose main reason to report for testing was an intention to receive treatment $25 \%$ were more likely to link to care $(\mathrm{AHR}=1.25 ; 95 \%$ CI 1.06 to 1.46$)$, table 2 .

\section{DISCUSSION}

This study prospectively measured linkage to care in remote and hard-to-reach areas and populations, and compared successful linkage and time to linkage into HIV care between two HIV testing service delivery 


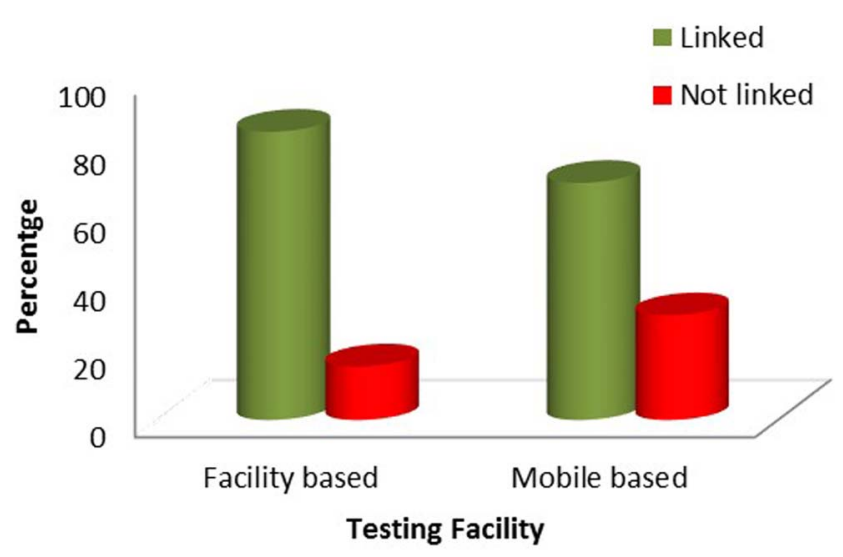

Figure 1 Linkage status.

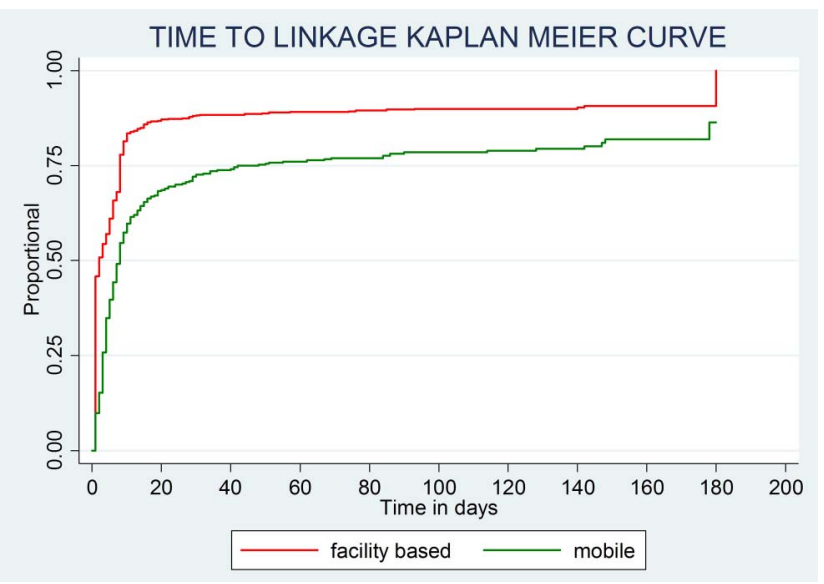

Figure 2 Survival analysis (KPM).

models in rural settings of the Mbeya Region in Tanzania.

The study was designed and implemented against the background of historically low rates of linkage to care, recent widespread implementation of mobile testing to address low population rates of HIV testing and evidence from other settings of significantly poorer linkage to care after HIV diagnosis at the mobile/outreachbased testing sites compared to facility-based testing sites.

Our study found that $78 \% \quad(\mathrm{n}=793)$ of individuals of the overall cohort had registered at CTCs within the first 6 months after diagnosis, representing a dramatic improvement in linkage to care after HIV diagnosis compared to the recent past in Tanzania. ${ }^{11-13}$

A number of studies on HIV testing and linkage to care in other SSA countries have reported linkage rates of more than $60 \% .^{15} 2126-29$ Our encouraging findings likely reflect a combination of health system and social changes, including reduction in stigma. Our study itself may also have increased linkage to care through regularly contacting and following up HIV-positive individuals.
Linkage to care in the group of people tested through the facility-based model was significantly higher compared to the group tested through the mobile/outreach services. More people were linked to care, and they linked modestly sooner in the health facility than mobile clinic arm. This aligns with earlier studies in Kenya, South Africa and systematic review and meta-analysis of community and facility-based HIV testing. ${ }^{15} 2129$

Likewise, a meta-analysis conducted in the USA on entry into medical care after HIV-positive diagnosis reported high entry by people testing at clinics and hospitals compared to other community testing settings. ${ }^{30}$

While the dramatic improvement in linkage across the overall cohort and the early linkage to the first step of care are encouraging findings, the continued gap in linkage to care between mobile-based and facility-based testing is important to address. It is possible that some of the respondents were lost to follow-up in the mobile/ outreach arm sought and were linked to care in other sites; however, we believe that significant health systemlevel barriers must be addressed to ensure timely linkage and, ultimately, retention in care.

Some of the outreach testing activities are performed very far from the clinics that offer CD4 testing and HIV care. For example, some clients in Chunya district must travel more than $100 \mathrm{~km}$ on a rough road to reach a facility that offers CD4 test services and ART. We suggest expansion of mobile staging and ART services in remote areas. Furthermore, healthcare providers should ensure that education and emphasis on the importance of being in HIV care, even if the client does not yet require ART according to local guidelines, are emphasised during counselling.

Disclosure of HIV sero-status to partners and/or family members was strongly associated with earlier linkage to care compared to those who did not disclose to partners, and/or family members/relatives, again corresponding with findings elsewhere ${ }^{31-33}$ and highlighting the continued importance of facilitating disclosure and social support.

We found that the majority of participants who reported, "Wanting to receive treatment in case they are infected with HIV" as one of the reasons for testing for HIV, tested at facility-based sites. This may suggest that they perceived themselves to be at higher risk, or that they already intended to seek care for their symptoms and that individuals testing at facilities were more willing to link immediately into care because they needed treatment. ${ }^{17}$ This would align with studies elsewhere that have reported higher CD4 counts at mobile sites than at facility-based sites; ${ }^{21}$ however, while we found slightly higher $\mathrm{CD} 4$ counts in the mobile testing arm, this difference was not statistically significant. We therefore think it is important to explore and address health system facilitators and barriers, such as the availability of integrated HIV testing, care and treatment services within the same facility/site.

This interpretation is supported by other findings from our study: a total of 265 individuals, $51.7 \%$, who 
Table 2 Factors associated with time to linkage at bivariate and Multivariate Cox regression

\begin{tabular}{|c|c|c|c|c|}
\hline Variable & Crude HR & $95 \% \mathrm{Cl}$ & Adjusted HR & $95 \% \mathrm{Cl}$ \\
\hline \multicolumn{5}{|l|}{ Gender } \\
\hline Male & Ref & & Ref & \\
\hline Female & 0.97 & 0.84 to 1.12 & 0.98 & 0.84 to 1.14 \\
\hline \multicolumn{5}{|l|}{ Age } \\
\hline $18-30$ & Ref & & Ref & \\
\hline $30-45$ & 0.95 & 0.81 to 1.11 & 0.98 & 0.83 to 1.17 \\
\hline $45-60$ & 1.18 & 0.95 to 1.47 & 1.12 & 0.87 to 1.44 \\
\hline$>60$ & 1.06 & 0.65 to 1.73 & 1.11 & 0.66 to 1.88 \\
\hline \multicolumn{5}{|l|}{ Marital status } \\
\hline Single & Ref & & Ref & \\
\hline Married & 1.24 & 0.98 to 1.56 & 1.06 & 0.83 to 1.35 \\
\hline Separated & 1.14 & 0.85 to 1.53 & 0.87 & 0.64 to 1.18 \\
\hline Divorced & 1.27 & 0.84 to 1.91 & 1.19 & 0.78 to 1.83 \\
\hline Widower & 1.37 & 1.02 to 1.83 & 1.15 & 0.82 to 1.61 \\
\hline \multicolumn{5}{|c|}{ Time to reach clinic (hours) } \\
\hline$<1$ & Ref & & Ref & \\
\hline $1-2$ & 1.06 & 0.89 to 1.25 & 1.03 & 0.86 to 1.22 \\
\hline $2-5$ & 0.97 & 0.74 to 1.28 & 1.17 & 0.88 to 1.55 \\
\hline$>5$ & 0.75 & 0.37 to 1.52 & 1.09 & 0.54 to 2.22 \\
\hline \multicolumn{5}{|l|}{ Testing site } \\
\hline Mobile based & Ref & & Ref & \\
\hline Facility based & 1.73 & 1.49 to $2.003^{*}$ & 1.78 & 1.53 to $2.07^{*}$ \\
\hline \multicolumn{5}{|c|}{ Health improved because of ARV } \\
\hline No & Ref & & Ref & \\
\hline Yes & 1.46 & 1.22 to $1.74^{*}$ & 1.01 & 0.82 to 1.24 \\
\hline \multicolumn{5}{|c|}{ Any friend/Family taking ARVs } \\
\hline No & Ref & & Ref & \\
\hline Yes & 1.35 & 1.16 to $1.58^{*}$ & 1.01 & 0.85 to 1.203 \\
\hline \multicolumn{5}{|c|}{ I want to receive treatment } \\
\hline No & Ref & & Ref & \\
\hline Yes & 1.25 & 1.07 to $1.45^{*}$ & 1.25 & 1.06 to $1.45^{*}$ \\
\hline \multicolumn{5}{|c|}{ Disclosure of HIV status } \\
\hline No & Ref & & Ref & \\
\hline Yes & 2.82 & 0.25 to $3.54^{*}$ & 2.64 & 2.05 to $3.39^{*}$ \\
\hline
\end{tabular}

tested at facility-based sites were able to link on the same day of HIV testing, while only $12 \%$ of those testing through the mobile/outreach model were able to link on the same day. This is likely associated with availability of HIV testing and HIV care and treatment services within the same compound at facility sites. Not surprisingly, some studies report that $\mathrm{CD} 4$ testing at the point of care reduces time for linkage, eligibility assessment and ART initiation,, ${ }^{34} 35$ and having HIV testing services and HIV care (CTC) at the same location improves rates of linkage to care and ART coverage. ${ }^{17} 36$ While Tanzania has made significant progress in increasing testing and linkage to care, our study strongly supports arguments for increasing the proportion of health facilities with care and treatment services from the current low level of $21.7 \% .^{13}$

Further analysis of our qualitative and quantitative data will help elucidate these findings. Nevertheless, studies on HIV testing indicate that outreach testing services increase access in remote areas, but linkage to care remains a problem. ${ }^{22} 37$ Our study supports these findings, while reporting significant improvements in overall linkage to care since 2009 and 2014. The strength of this study is that we had a large sample of newly HIV-positive diagnosed individuals in the cohort, enrolled from 16 different sites who were followed up for 6 months from the time of diagnosis. The project team used telephone calls to follow-up clients on their dates of next visit to clinic. Use of phone calls may have been one of the factors that facilitated or enhanced linkage to care among the study participants.

The study has some limitations. We were not able to see all clients during the study period, despite efforts to track them through telephone calls by study team, CTC and community-based healthcare providers in their respective areas. It may be assumed that the clients might have moved to other places due to prevailing trade routes with extensive cross-border migration; however, our study was not able to ascertain the exact name of linkage site and linkage beyond the study sites. 
This warrants further investigation. An additional limitation of our study is that the random selection of facilitybased sites yielded a sample where all facilities had on-site CTCs.

\section{CONCLUSIONS}

Linkage to care is the bridge between HIV testing and treatment/care services for HIV-positive individuals. In comparison with previous studies conducted in 2009, 2012 and 2014 in Tanzania, this study shows that significantly more newly diagnosed HIV-positive individuals had linked to care within a short time of testing. We also found that linkage to care within 6 months of HIV testing was significantly higher from health facility-based HIV-testing sites compared to mobile/outreach sites. Finally, though of more modest clinical and population health significance, these individuals were linked into care significantly sooner, particularly at sites where the same-day registration for care and treatment was possible. Individuals who had disclosed their HIV status to their partner and/or family members were more likely to link to care earlier than those who did not disclose to anyone. Findings from this study suggest that although mobile/outreach service delivery models bring HIV testing services closer to people in remote and resource-restrained areas, there is still a significant gap in timely linkage to HIV care compared to sites within established health facilities. Thus, strategies that are more effective are needed to further improve linkage through this model of service delivery, including increased attention to effectively communicating the importance of linkage to care even for people who do not feel sick. In addition, the availability of care and treatment at facility-based testing sites should be significantly increased from the current low levels of $<21.7 \%$ of public facility-based testing sites offering treatment and care.

\section{Author affiliations \\ ${ }^{1}$ NIMR-Mbeya Medical Research Centre (MMRC), Mbeya, Tanzania \\ ${ }^{2}$ School of Public Health, University of Western Cape, Cape Town, South Africa \\ ${ }^{3}$ School of Public Health, Mekelle University, Mekelle, Ethiopia \\ ${ }^{4}$ National Institute for Medical Research (NIMR), Dar es Salaam, Tanzania \\ ${ }^{5}$ Division of Infectious Diseases and Tropical Medicine, Medical Centre of the University of Munich (LMU), Munich, Germany \\ ${ }^{6}$ University of Montreal Hospital Research Centre and School of Public Health, Université de Montréal, Montreal, Quebec, Canada}

Acknowledgements The authors are very thankful to all study participants and dedicated research assistants, namely Jane Merere, Philmin John, Nestory Sidinda, Aloyce Kabuche, Elesia Kaduma, Obeth Emmanuel, Mariam Paul, Benson Msomba and data entry team at MRCC for special cooperation that facilitated data collection and processing. The especially thank MMRC Mobile Diagnostic and Training Centre (MDTC) project coordinators and all healthcare providers at the study sites and the home-based cares (HBCs) for their hard work, and HJF-Global Health International for funding the MDTC. The authors thank the Regional Medical Officer (RMO), the District Medical officers (DMOs), the District AIDS Control Coordinators (DACC) and the facility/site in-charges/managers for their continued cooperation and support. The authors thanks the University of Western Cape (UWC) for approving the study and supervision. ESS thanks the Institute of Tropical Medicine-Antwerp for the opportunity of a writing retreat at ITM-Antwerp through the SIPHI scholarship. The authors thank the management of Mbeya Medical Research Centre (MMRC) and the African Doctoral Dissertation Research Fellowship (ADDRF) Award 2014-2016-ADF 009, offered by the African Population and Health Research Centre (APHRC) in partnership with the International Development Research Centre (IDRC) for financing and supporting the study.

Contributors ESS designed the study, collected and assembled the data, and participated in data analysis and interpretation. ESS, PC, and LM, drafted the article. CZ, AKM and WL were study supervisors and critically revised the article. ESS and WO analyzed and interpreted the data.

Funding Social Innovation in Public Health Impulse (SIPHI) fellowship through the University of Western Cape (PhD writing sabbatical); NIMR-Mbeya Medical Research Centre (7500\$); African Doctoral Dissertation Research Fellowship (ADDRF) award 2014, offered by the African Population and Health Research Centre (APHRC) in partnership with the International Development Research Centre (IDRC) (2014 Award).

Disclaimer This manuscript is published with permission from Director General, National Institute for Medical Research.

Competing interests None declared.

Patient consent Obtained.

Ethics approval This study was approved by the Medical Research Coordinating Committee of the National Institute for Medical Research also was approved by the Senate Research Committee of the University of Western Cape

Provenance and peer review Not commissioned; externally peer reviewed.

Data sharing statement Extra data can be accessed via the Dryad data repository at http://datadryad.org/ with the doi:10.5061/dryad.4g0vt

Open Access This is an Open Access article distributed in accordance with the Creative Commons Attribution Non Commercial (CC BY-NC 4.0) license, which permits others to distribute, remix, adapt, build upon this work noncommercially, and license their derivative works on different terms, provided the original work is properly cited and the use is non-commercial. See: http:// creativecommons.org/licenses/by-nc/4.0/

\section{REFERENCES}

1. AmfAR. "amfAR-Statistics," Statistics. World Wide. Sub Saharan Africa, 2015. http://www.amfar.org/worldwide-aids-stats/ (accessed 01 Aug 2015).

2. Elul B, Lahuerta M, Abacassamo $F$, et al. A combination strategy for enhancing linkage to and retention in HIV care among adults newly diagnosed with HIV in Mozambique: study protocol for a site-randomized implementation science study. BMC Infect Dis 2014;14:549.

3. Naik R, Doherty $T$, Jackson $\mathrm{D}$, et al. Linkage to care following a home-based HIV counselling and testing intervention in rural South Africa. J Int AIDS Soc 2015;18:19843.

4. Gerdts SE, Wagenaar BH, Micek MA, et al. Linkage to HIV care and antiretroviral therapy by HIV testing service type in central Mozambique: a retrospective cohort study. J Acquir Immune Defic Syndr 2014;66:e37-44.

5. MRHR. Mbeya regional hospital report. Mbeya, Tanzania, 2010

6. MRACP. Mbeya Regional AIDS Control Program: Annual Report 2012.

7. del Rio C. Cascade of care and its relevance to seek, test, treat and retain strategy. Emory Center for AIDS Research, 2011.

8. Kranzer K, Zeinecker J, Ginsberg P, et al. Linkage to HIV care and antiretroviral therapy in Cape Town, South Africa. PLoS One 2010;5:1-6.

9. Suthar AB, Ford N, Bachanas PJ, et al. Towards universal voluntary HIV testing and counselling: a systematic review and meta-analysis of community-based approaches. PLoS Med 2013;10:e1001496.

10. Ministry of Health and Social Welfare (MOHsw). National Guideline for Management of HIV and AIDS, 5th eddition, Circular of Revised guideline for management of HIVIAIDS. 2015.

11. Nsigaye R, Wringe A, Roura M, et al. From HIV diagnosis to treatment: evaluation of a referral system to promote and monitor 
access to antiretroviral therapy in rural Tanzania. $J$ Int AIDS Soc 2009;12:31.

12. Simmelink AM. High uptake but low rates of linkage to care following home-based integrated HIV voluntary counseling and testing services and non-communicable disease screening in Ifakara, Tanzania. MZIMA open adult health community cohort. 2014.

13. MRACP. Mbeya Regional AIDS Control Program: Annual Report. 2014.

14. Mugavero MJ, Norton WE, Saag MS. Health care system and policy factors influencing engagement in HIV medical care: piecing together the fragments of a fractured health care delivery system. Clin Infect Dis 2011;52(Suppl 2):S238-46.

15. Genberg BL, Naanyu V, Wachira J, et al. Linkage to and engagement in HIV care in western Kenya: an observational study using population-based estimates from home-based counselling and testing. Lancet HIV 2015;2:e20-6.

16. Leon N, Mathews $\mathrm{C}$, Lewin $\mathrm{S}$, et al. A comparison of linkage to HIV care after provider-initiated HIV testing and counselling (PITC) versus voluntary HIV counselling and testing (VCT) for patients with sexually transmitted infections in Cape Town, South Africa. BMC Health Serv Res 2014;14:350.

17. Layer EH, Kennedy CE, Beckham SW, et al., LTC Tanzania Collaborative Study Team. Multi-level factors affecting entry into and engagement in the HIV continuum of care in Iringa, Tanzania. PLoS One 2014:9:e104961.

18. MacPherson P, MacPherson EE, Mwale D, et al. Barriers and facilitators to linkage to ART in primary care: a qualitative study of patients and providers in Blantyre, Malawi. J Int AIDS Soc 2012;15:1-10.

19. Govindasamy D, Ford N, Kranzer K. Risk factors, barriers and facilitators for linkage to antiretroviral therapy care: a systematic review. AIDS 2012;26:2059-67.

20. NACP, National AIDS Control Program in Tanzania, 2012.

21. Bassett IV, Regan S, Luthuli $P$, et al. Linkage to care following community-based mobile HIV testing compared with clinic-based testing in Umlazi Township, Durban, South Africa. Hiv Med 2014;15:367-72.

22. Labhardt ND, Motlomelo M, Cerutti B, et al. Home-based versus mobile clinic HIV testing and counseling in rural Lesotho: a cluster-randomized trial. PLoS Med 2014;11:e1001768.

23. Holzemer WL, Uys L, Makoae L, et al. A conceptual model of HIV/ AIDS stigma from five African countries. $J$ Adv Nurs 2007;58:541-51.
24. The National Bureau of Statistics-Tanzania: Population and Housing Census Population Distribution by 2013.

25. Rosen S, Fox MP. Retention in HIV care between testing and treatment in Sub-Saharan Africa: a systematic review. PLoS Med 2011;8:e1001056.

26. Muhula S, Memiah P, Mbau L, et al. Uptake and linkage into care over one year of providing HIV testing and counselling through community and health facility testing modalities in urban informal settlement of Kibera, Nairobi Kenya. BMC Public Health 2016;16:373.

27. Hatcher AM, Kwena Z, Johnson MO. Counseling and testing in rural Kenya. AIDS Behav 2015;16:1295-307.

28. Rasschaert F, Decroo T, Remartinez D, et al. Adapting a community-based ART delivery model to the patients' needs: a mixed methods research in Tete, Mozambique. BMC Public Health 2014;14:364.

29. Sharma M, Ying R, Tarr G, et al. A systematic review and meta-analysis of community and facility-based approaches to address gaps in HIV testing and linkage in sub-Saharan Africa. HHS Public Access 2015;2:1-26.

30. Marks G, Gardner LI, Craw J, et al. Entry and retention in medical care among HIV-diagnosed persons: a meta-analysis. AIDS 2014;24:2665-78

31. Ostermann J, Pence B, Whetten K, et al. HIV serostatus disclosure in the treatment cascade: evidence from Northern Tanzania. AIDS Care 2015;27(Suppl 1):59-64.

32. Hodgson I, Plummer ML, Konopka SN, et al. A systematic review of individual and contextual factors affecting ART initiation, adherence, and retention for HIV-infected pregnant and postpartum women. PLoS One 2014;9:e111421.

33. Dima AL, Stutterheim SE, Lyimo R, et al. Advancing methodology in the study of HIV status disclosure: The importance of considering disclosure target and intent. Soc Sci Med 2014;108:166-74.

34. Wynberg E, Cooke G, Shroufi A, et al. Impact of point-of-care CD4 testing on linkage to HIV care: a systematic review. J Int AIDS Soc 2014;17:18809.

35. Hyle EP, Jani IV, Lehe J, et al. The clinical and economic impact of point-of-care CD4 testing in Mozambique and other resource-limited settings: a cost-effectiveness analysis. PLoS Med 2014;11:e1001725.

36. Wachira J, Naanyu V, Genberg B, et al. Health facility barriers to HIV linkage and retention in Western Kenya. BMC Heal Serv Res 2014;14:646.

37. Kranzer K, Zeinecker J, Ginsberg P, et al. Linkage to HIV care and antiretroviral therapy in Cap Town, South Africa. PLoS One 2010;5: e13801. 